\section{PWE-58 ARE NOVEL AND ESTABLISHED ANORECTAL PHYSIOLOGY DIAGNOSTICS ASSOCIATED WITH SYMPTOMS AND QUALITY-OF-LIFE IN FAECAL INCONTINENCE?}

Caroline Byrne*, Dipesh Vasant, Edward Kiff, Peter Whorwell, Abhiram Sharma, Karen Telford. Manchester University NHS Foundation Trust, Manchester, UK

10.1136/gutjnl-2021-BSG.320

Introduction High-resolution anorectal manometry (HRAM) is an established assessment modality. Novel techniques, such as the Functional lumen imaging probe (EndoFLIP) and Anal Acoustic Reflectometry (AAR) are currently predominantly considered research tools. However, there is recognised, and well-documented, discordance between HRAM parameters, symptom severity and quality-of-life scores (QoL) in patients with faecal incontinence (FI). The aim of this study was to examine metrics from each of these three modalities and investigate whether they correlated with patients' symptoms and QoL.

Methods Females with FI undergoing HRAM at a tertiary pelvic floor centre were recruited and completed a series of symptom questionnaires including Vaizey FI score (VFI), Constipation Scoring System (CSS), and the Manchester Health QoL (MHQ). Following HRAM, patients had AAR and FLIP subsequently in a randomised order. Resting and squeeze parameters were recorded (Opening/Squeeze opening pressure $(\mathrm{Op} / \mathrm{SqOp})$ for AAR, distensibility index (DI) for EndoFLIP and resting/incremental squeeze pressure for HRAM). Correlations between symptom scores and QoL measures were compared with anorectal physiology metrics using Spearman's correlation.

Results Twenty females [median age 61.5 years (IQR 51.566yrs)] with FI (mixed 45\%, passive 35\%, urge 20\%) were recruited. There was no difference in median VFI $(p=0.293)$, CSS $(p=0.473)$ or MHQ $(p=0.490)$ scores between FI subtype. Those with a low resting and incremental squeeze pressures (HRAM) had a higher Vaizey score and reported a poorer QoL (coefficient -0.46, p $=0.046$ and $-0.58, p=0.09$ respectively). The higher the DI during squeeze and lower the squeeze opening pressure, the higher the Vaizey score (coefficient $0.51, \mathrm{p}=0.028$ and -0.49 and $\mathrm{p}=0.034$ respectively) (Table 1).

Conclusions This novel study comparing metrics from three anorectal physiology modalities has demonstrated that each technique had at least one parameter (resting or squeeze) that correlated with either symptom severity and QoL in patients with FI, supporting the need for further research on the utility of EndoFLIP and AAR.

\section{PWE-59 PATIENT ACCEPTANCE OF ANORECTAL PHYSIOLOGY DIAGNOSTICS: HOW IMPORTANT IS THE CATHETER?}

Caroline Byrne*, Dipesh Vasant, Edward Kiff, Peter Whorwell, Abhiram Sharma, Karen Telford. Manchester University NHS Foundation Trust, Manchester, UK

\subsection{6/gutjnl-2021-BSG.321}

Introduction The diameter of high-resolution anorectal manometry (HRAM) catheters vary from $2.6 \mathrm{~mm}$ up to $10.75 \mathrm{~mm}$ for some high-definition devices. Other catheter-based physiology assessment tools such as the Functional lumen imaging probe (FLIP) use of a probe with a starting diameter of $3 \mathrm{~mm}$. Anal acoustic reflectometry (AAR) however is a 'catheter-less' technique as it does not distort the anal canal due to the extremely thin (diameter $0.71 \mathrm{~mm}$ ) flexible probe occupying a small cross-sectional area $\left(0.4 \mathrm{~mm}^{2}\right)$. Therefore the primary aim of this study was to explore which system patients found more acceptable.

Methods Females with faecal incontinence (FI) symptoms attending for HRAM at a tertiary pelvic floor centre were recruited. Following HRAM, patients underwent AAR or FLIP in a randomised order. Resting and squeeze parameters were recorded for each modality and at the end of all 3 tests, patients completed a visual analogue scale independent of the researcher $[($ scale $0-10) \quad 0=$ no pain/acceptable test, $10=$ severe discomfort/an unacceptable test].

Results Twenty females [median age 61.5 years (IQR 51.566yrs)] with varying sub-types of FI (mixed 45\%, passive $35 \%$, urge 20\%) were recruited. Physiology parameters and VAS scores are presented in table 1. All of the respective median resting/squeeze parameters, [except for incremental squeeze pressure (HRAM)], for each modality were below laboratory normative values. Eleven patients (55\%) reported equal acceptability for all 3 tests, scoring either $0 / 10$ or $1 / 10$ for each. Median scores for all three modalities were low $(0.5 / 10-1 / 10)$.

Conclusions This study has shown for the first time that the majority of patients found that all 3 modalities were of equal acceptability with minimal discomfort. It is acknowledged that

Abstract PWE-58 Table 1 Physiology parameters and correlations with symptom severity and QoL questionnaires (Spearman's correlation, $r_{s}$ )

\begin{tabular}{|c|c|c|c|c|c|c|c|c|}
\hline & Median resting & VFI & CSS & MHQ & \multirow{2}{*}{$\begin{array}{l}\text { Median squeeze } \\
\text {-result } \\
\text { (IQR) }\end{array}$} & \multirow{2}{*}{$\begin{array}{l}\text { VFI } \\
r_{s} \\
\text { (p-value) }\end{array}$} & \multirow[t]{2}{*}{ CSS } & \multirow[t]{2}{*}{ MHQ } \\
\hline & $\begin{array}{l}\text { result } \\
(\mathrm{IQR})\end{array}$ & $\begin{array}{l}r_{s} \\
\text { (p-value) }\end{array}$ & & & & & & \\
\hline HRAM & 40.04 & -0.46 & 0.26 & -0.26 & 81.4 & -0.32 & 0.96 & -0.58 \\
\hline (Resting/ & $(25.6-6.5)$ & $(0.046)$ & $(0.288)$ & $(0.281)$ & (54.4-147) & $(0.177)$ & $(0.697)$ & $(0.009)$ \\
\hline $\begin{array}{l}\text { Incremental Squeeze pressure, } \\
\left.\mathrm{CmH}_{2} \mathrm{O}\right)\end{array}$ & & * & & & & & & * \\
\hline FLIP & 1.6 & 0.35 & -0.20 & 0.15 & 0.7 & 0.51 & -0.22 & 0.33 \\
\hline$\left(\mathrm{DI}, \mathrm{mm}^{2} / \mathrm{mmHg}\right)$ & $(0.8-1.7)$ & $(0.146)$ & $(0.404)$ & $(0.555)$ & $(0.5-1.6)$ & $\begin{array}{l}(0.028) \\
*\end{array}$ & $(0.358)$ & $(0.174)$ \\
\hline AAR & 41.4 & -0.40 & 0.18 & -0.10 & 79.8 & -0.49 & 0.414 & -0.23 \\
\hline$\left(\mathrm{Op} / \mathrm{SqOp}, \mathrm{cmH}_{2} \mathrm{O}\right)$ & $(25.1-63.1)$ & $(0.093)$ & $(0.456)$ & $(0.673)$ & (46.1-124.9) & $\begin{array}{l}(0.034) \\
*\end{array}$ & $(0.078)$ & $(0.344)$ \\
\hline
\end{tabular}




\begin{tabular}{|c|c|c|c|c|c|}
\hline $\begin{array}{l}\text { Modality } \\
\text { (parameters recorded, } \\
\text { unit) } n=20\end{array}$ & $\begin{array}{l}\text { Median } \\
\text { resting } \\
\text { result } \\
\text { (IQR) }\end{array}$ & $\begin{array}{l}\text { Abnormal/ } \\
\text { Normal }\end{array}$ & $\begin{array}{l}\text { Median } \\
\text { squeeze } \\
\text { result } \\
\text { (IQR) }\end{array}$ & $\begin{array}{l}\text { Abnormal/ } \\
\text { Normal }\end{array}$ & $\begin{array}{l}\text { Median } \\
\text { VAS } \\
\text { score } \\
(I Q R)\end{array}$ \\
\hline $\begin{array}{l}\text { HRAM } \\
\text { (Resting/Incremental } \\
\text { Squeeze pressure, } \\
\mathrm{CmH}_{2} \mathrm{O} \text { ) }\end{array}$ & $\begin{array}{l}40.04 \\
(25.6- \\
76.5)\end{array}$ & Abnormal & $\begin{array}{l}81.4 \\
(54.4- \\
147.0)\end{array}$ & Normal & $\begin{array}{l}1 \\
(0- \\
2.8)\end{array}$ \\
\hline $\begin{array}{l}\text { FLIP } \\
\text { (Distensibility index, } \\
\mathrm{mm}^{2} / \mathrm{mmHg} \text { ) }\end{array}$ & $\begin{array}{l}1.6 \\
(0.8-1.7)\end{array}$ & Abnormal & $\begin{array}{l}0.7 \\
(0.5-1.6)\end{array}$ & Abnormal & $\begin{array}{l}0 \\
(0-1)\end{array}$ \\
\hline $\begin{array}{l}\text { AAR } \\
\text { (Opening/Squeeze } \\
\text { opening pressure, } \\
\mathrm{CmH}_{2} \mathrm{O} \text { ) }\end{array}$ & $\begin{array}{l}41.4 \\
(25.1- \\
63.1)\end{array}$ & Abnormal & $\begin{array}{l}79.8 \\
(46.1- \\
124.9)\end{array}$ & Abnormal & $\begin{array}{l}0.5 \\
(0-1)\end{array}$ \\
\hline
\end{tabular}

assessment of anorectal function is crucial in those with FI however, fear of tests/investigations can be a barrier to accessing services. Further qualitative research on patients perceptions/preferences of anorectal investigations and treatments will help to provide education for health practitioners and service users, and hopefully improve the patient journey experience.

\section{PWE-60 UNDIAGNOSED PSYCHOPATHOLOGY IS PREVALENT AND SIGNIFICANT IN PATIENTS ATTENDING A TERTIARY NEUROGASTROENTEROLOGY CLINIC}

${ }^{1}$ Alicia Green, ${ }^{1}$ Inga Daugirdaite*, ${ }^{1,2}$ Qasim Aziz, ${ }^{1,2}$ Asma Fikree. 'Wingate Institute of Neurogastroenterology, London, UK; ${ }^{2}$ Barts Health NHS Trust, London, UK

\subsection{6/gutjpl-2021-BSG.322}

Introduction According to the biopsychosocial model for functional gastrointestinal (GI) disorders, the presence of psychopathology influences symptom presentation and should be targeted in the management plan. We aimed to determine what proportion of patients attending a tertiary neurogastroenterology clinic had evidence of anxiety, depression and eating disorders which had not been previously identified, but which may be very important for treatment strategies.

Methods Consecutive patients attending a tertiary neurogastroenterology clinic completed validated questionnaires to screen for anxiety and depression (HADS-A/D $>11$ ), eating disorders $(\mathrm{SCOFF}>=2)$, avoidant restrictive eating disorders (ARFID using NIAS > 28), personality disorders (SAPAS > 2), GI symptoms (GSRS), visceral sensitivity index (VSI) and quality of life (QOL using SF36). They were asked specifically about a history of anxiety, depression and eating disorders. Questionnaire scores were compared using a Mann Whitney U test - in view of the multiple comparisons a $\mathrm{p}$ value $<0.01$ was considered significant.

Results 186 patients (aged 16-84, 85\% female) completed the questionnaires. 41 patients (22\%) screened positive for depression and $73(39 \%)$ for anxiety, though a third of each of these did not document a diagnosis of depression or anxiety. $22 \%$ screened positive for an eating disorder and $25 \%$ for ARFID, although only 16\% documented a diagnosis of an eating disorder. $48 \%$ screened positive for a personality disorder. Patients who screened positive for depression and anxiety had

\section{Abstract PWE-60 Table 1}

\begin{tabular}{|c|c|c|c|c|c|c|}
\hline & $\begin{array}{l}\text { Negative } \\
\text { depression } \\
\text { screen }\end{array}$ & $\begin{array}{l}\text { Positive } \\
\text { depression } \\
\text { screen }\end{array}$ & $P$ value & $\begin{array}{l}\text { Negative } \\
\text { anxiety } \\
\text { screen }\end{array}$ & $\begin{array}{l}\text { Positive } \\
\text { anxiety } \\
\text { screen }\end{array}$ & $P$ value \\
\hline $\begin{array}{l}\text { Mean overall } \\
\text { GSRS }\end{array}$ & $3.54 \pm 1.10$ & $4.21 \pm 1.11$ & 0.001 & $\begin{array}{l}3.47 \pm \\
1.06\end{array}$ & $\begin{array}{l}4.03 \pm \\
1.18\end{array}$ & 0.001 \\
\hline $\begin{array}{l}\text { Mean GSRS } \\
\text { abdo pain }\end{array}$ & $3.78 \pm 1.27$ & $4.75 \pm 1.19$ & $<0.0001$ & $\begin{array}{l}3.78 \pm \\
1.33\end{array}$ & $\begin{array}{l}4.34 \pm \\
1.22\end{array}$ & 0.005 \\
\hline $\begin{array}{l}\text { Mean GSRS } \\
\text { reflux }\end{array}$ & $3.19 \pm 1.90$ & $3.87 \pm 2.00$ & 0.06 & $\begin{array}{l}3.16 \pm \\
1.95\end{array}$ & $\begin{array}{l}3.63 \pm \\
1.89\end{array}$ & 0.086 \\
\hline $\begin{array}{l}\text { Mean GSRS } \\
\text { indigestion }\end{array}$ & $3.82 \pm 1.51$ & $4.19 \pm 1.53$ & 0.204 & $\begin{array}{l}3.67 \pm \\
1.52\end{array}$ & $\begin{array}{l}4.26 \pm \\
1.46\end{array}$ & 0.01 \\
\hline $\begin{array}{l}\text { Mean GSRS } \\
\text { diarrhoea }\end{array}$ & $3.01 \pm 1.86$ & $4.08 \pm 1.79$ & 0.001 & $\begin{array}{l}2.96 \pm \\
1.84\end{array}$ & $\begin{array}{l}3.69 \pm \\
1.90\end{array}$ & 0.01 \\
\hline $\begin{array}{l}\text { Mean GSRS } \\
\text { constipation }\end{array}$ & $3.70 \pm 1.97$ & $4.15 \pm 1.94$ & 0.213 & $\begin{array}{l}3.66 \pm \\
1.93\end{array}$ & $\begin{array}{l}4.02 \pm \\
2.01\end{array}$ & 0.238 \\
\hline $\begin{array}{l}\text { SF36 General } \\
\text { health }\end{array}$ & $\begin{array}{l}30.85 \pm \\
19.60\end{array}$ & $\begin{array}{l}14.05 \pm \\
10.78\end{array}$ & $<0.0001$ & $\begin{array}{l}31.31 \pm \\
19.77\end{array}$ & $\begin{array}{l}20.35 \pm \\
16.56\end{array}$ & $<0.0001$ \\
\hline $\begin{array}{l}\text { SF36 Physical } \\
\text { functioning }\end{array}$ & $\begin{array}{l}51.50 \pm \\
34.04\end{array}$ & $\begin{array}{l}27.50 \pm \\
27.26\end{array}$ & $<0.0001$ & $\begin{array}{l}51.14 \pm \\
34.52\end{array}$ & $\begin{array}{l}38.06 \pm \\
32.03\end{array}$ & 0.007 \\
\hline $\begin{array}{l}\text { SF36 Social } \\
\text { functioning }\end{array}$ & $\begin{array}{l}47.25 \pm \\
30.66\end{array}$ & $\begin{array}{l}16.07 \pm \\
21.61\end{array}$ & $<0.0001$ & $\begin{array}{l}48.54 \pm \\
31.16\end{array}$ & $\begin{array}{l}27.08 \pm \\
27.91\end{array}$ & $<0.0001$ \\
\hline $\begin{array}{l}\text { SF36 Energy/ } \\
\text { fatigue }\end{array}$ & $\begin{array}{l}31.06 \pm \\
20.84\end{array}$ & $\begin{array}{l}12.02 \pm \\
26.69\end{array}$ & 0.001 & $\begin{array}{l}32.61 \pm \\
21.98\end{array}$ & $\begin{array}{l}17.57 \pm \\
15.83\end{array}$ & $<0.0001$ \\
\hline VSI score & $\begin{array}{l}36.11 \pm \\
18.29\end{array}$ & $\begin{array}{l}45.41 \pm \\
20.60\end{array}$ & 0.006 & $\begin{array}{l}34.81 \pm \\
18.99\end{array}$ & $\begin{array}{l}43.36 \pm \\
18.56\end{array}$ & 0.006 \\
\hline
\end{tabular}

more severe GI symptoms, particularly pain, higher VSI and worse QOL (Table 1).

Discussion/conclusion Simple questionnaire screening identifies a high prevalence of psychopathology in patients attending neurogastroenterology clinics, a third of whom had no documented diagnosis of psychopathology, so this would have been missed by simple history taking. Patients who screen positive for psychopathology have more GI symptoms and worse QOL so it is important to identify this as addressing it may improve GI symptoms. Having a dedicated gastro-psychologist in neurogastroenterology centres would help with screening and managing these difficult conditions yet the majority of UK centres do not have access to this.

\section{PWE-61 AN EARLY EVALUATION OF PARTICIPANT RECRUITMENT AND COMPLIANCE FOLLOWING THE VIRTUALISATION OF RELIEVE IBS-D TRIAL}

${ }^{1}$ Cho Ee $\mathrm{Ng}^{*},{ }^{2} \mathrm{C}$ Howell, ${ }^{2} \mathrm{~A}$ Kemppinen, ${ }^{3} \mathrm{H}$ Forsyth, ${ }^{3} \mathrm{G}$ Richell, ${ }^{2} \mathrm{E}$ Markaryan, ${ }^{4} \mathrm{~J}$ McLaughlin, ${ }^{5} \mathrm{C}$ Knowles, ${ }^{1,3}$ Yan Yiannakou. ${ }^{1}$ University Hospital North Durham, Durham, UK; ${ }^{2}$ EnteroMed Ltd, London, UK; ${ }^{3}$ NIHR patient recruitment centre, Newcastle, UK; ${ }^{4}$ University of Manchester, Manchester, UK; ${ }^{5}$ Queen Mary University of London, London, UK

\subsection{6/gutjnl-2021-BSG.323}

Introduction Irritable bowel syndrome (IBS) is a common condition but recruitment to IBS clinical trials has been challenging due to non-specialist follow-up, poor diagnostic coding, and complexity of ROME IV criteria. The RELIEVE-IBSD study was delivered partly as a traditional site-dependent trial (SDT) and a remote virtual trial (VT). We aimed to compare 